\title{
Grau de bem-estar relativo de frangos em diferentes densidades de lotação
}

\author{
[Broiler welfare in relation to stocking density] \\ A. Bonamigo $^{1}$, C.B.S. Silva ${ }^{2}$, C.F.M. Molento ${ }^{2}$ \\ ${ }^{1}$ Pós-graduação - UFPR - Curitiba, PR \\ ${ }^{2}$ Laboratório de Bem-estar Animal - UFPR - Curitiba, PR
}

\begin{abstract}
RESUMO
Avaliou-se o impacto da redução da densidade de lotação sobre o bem-estar animal (BEA) de frangas de corte, produzidas para abate precoce. Para isso, aos 12 dias de idade, 800 frangas, comerciais, foram distribuídas em baias de $4 \mathrm{~m}^{2}$, sendo oito com 10 aves $(17,0 \mathrm{~kg}) / \mathrm{m}^{2}$ (DL10) e oito com 15 aves $(25,5 \mathrm{~kg}) / \mathrm{m}^{2}$ (DL15). As condições ambientais foram avaliadas utilizando-se o índice de temperatura e umidade (ITU). Para avaliação de bem-estar, foi construído um etograma; hemograma e testes bioquímicos séricos, desempenho zootécnico e problemas locomotores foram avaliados. Em 43,6\% do tempo, o ITU ficou acima do recomendado, e não foram observadas diferenças comportamentais. Diferenças significativas

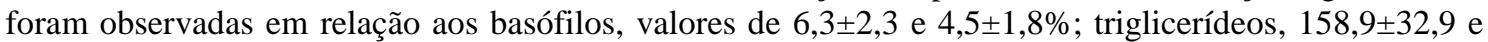
$186,3 \pm 43,9 \mathrm{mg} / \mathrm{dL}$; globulinas, $1,8 \pm 0,5$ e $1,5 \pm 0,4 \%$. Menos peso final ocorreu em DL15, $1,65 \pm 0,28 \mathrm{~kg}$, $1,69 \pm 0,13$. Problemas locomotores não diferiram entre tratamento. A consideração conjunta de menor peso final, maiores taxas de triglicerídeos e menores valores de globulinas sugere menor grau de bemestar em DL15. Índices de ITU e relação heterófilo:linfócito acima do normal sugerem baixo grau de bem-estar em ambas as densidades.
\end{abstract}

Palavras-chave: franga de corte, desempenho, hemograma, índice heterófilo:linfócito

\begin{abstract}
The objective was to evaluate the impact of reducing box density on female broiler welfare (BEA). At 12 days of age 800 females Cobb-500 ${ }^{\circledR}$ were weighed and distributed into randomized boxes of $4 m^{2}$, being eight boxes with 10 birds $(17,0 \mathrm{~kg}) / \mathrm{m}^{2}$ (DL10) and eight with $15 \mathrm{birds}(25,5 \mathrm{~kg}) / \mathrm{m}^{2}$ (DL15). Environmental conditions were evaluated using the temperature and humidity index (ITU). Hematologic and biochemical tests were performed. Production data was registered and locomotion problems were evaluated. It was observed that for $43 \%$ of the time the ITU was above the recommendations. Statistical differences were observed in relation to basophils $(6,3 \pm 2,3$ and 4,5 $\pm 1,8 \%)$, triglycerides $(158,9 \pm 32,9$ e 186,3 $\pm 43,9 \mathrm{mg} / \mathrm{dl})$,

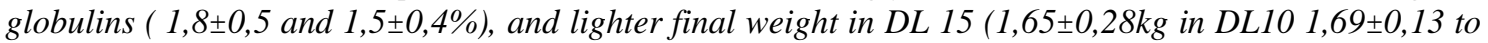
DL 15.) No difference in locomotion problems was observed. Results of lighter final weight, higher value of triglycerides, and lower value of globulins suggest worse welfare in DL 15. ITU above recommendation and high values of H:L suggest low welfare degree in both densities.
\end{abstract}

Keyword: pullet, production indices, hematology, heterophil/lymphocyte index

\section{INTRODUÇÃO}

A densidade de lotação de aves no sistema intensivo de produção é uma questão polêmica intensamente debatida. Em relação ao bem-estar animal, vários estudos relacionam os problemas oriundos do aumento de densidade, sendo que parte deles demonstra prejuízos diretos à produção, como queda no ganho de peso e no rendimento de carcaça, aumento de problemas de pele, diminuição da uniformidade do lote e aumento de mortalidade (Martrenchar et al., 1997; Dozier et al., 2005). Outros estudos

Recebido em 18 de outubro de 2010

Aceito em 12 de agosto de 2011

E-mail: abonamigo@yahoo.com.br 
comprovam uma queda no grau de bem-estar do indivíduo, podendo ser observados problemas de andadura, aumento de dermatites de coxim plantar, artrites, escoriações e feridas de pele, alterações comportamentais e fisiológicas, como o aumento dos níveis de proteínas, glicose e triglicerídeos na corrente sanguínea (Puvadolpirod e Thaxton, 2000a) e alteração nas células sanguíneas, com variação na relação entre o número de heterófilos e linfócitos, sendo considerados ótimos indicadores de estresse crônico (Gross e Siegel, 1993).

Mediante o conhecimento desses problemas e as pressões sociais em defesa do bem-estar animal, leis, normas e diretrizes vêm sendo criadas para estipular limites para as práticas de produção, inclusive para densidade de lotação. Tais limites, além de promoverem a saúde e o bem-estar animal, nivelam as práticas de manejo e contribuem para a manutenção da competitividade dentro do setor avícola (Bessei, 2006). Porém, segundo Estevez (2007), a precisão científica necessária para estabelecimento de um limite de bem-estar nem sempre é fácil de ser alcançada, pois existe um decréscimo natural de bem-estar e saúde associado ao aumento da idade do animal; à diferença entre indicadores e conceitos de saúde e bem-estar; entre sistemas de produção e linhagens; e entre os achados em experimentos controlados e situações reais de produção comercial.

Por tais motivos, pode-se observar variação entre as exigências de cada sociedade e suas associações representantes. Como exemplo, a Royal Society for the Prevendion of Cruelty to Animals (RSPCA..., 2008) estabeleceu um máximo de $30 \mathrm{~kg} / \mathrm{m}^{2}$ ou 19 aves $/ \mathrm{m}^{2}$; a Assured Chicken Production (Poultry..., 2009) estipula $25 \mathrm{~kg} / \mathrm{m}^{2}$ para certificação. No Brasil, o protocolo de bem-estar de frangos e perus publicado pela União Brasileira da Avicultura (Protocolo..., 2008) estipula o limite de $39 \mathrm{~kg} / \mathrm{m}^{2}$.

Além da quantidade de quilogramas por metro quadrado, é importante observar que nem todos os limites estipulados citam o número máximo aceitável de aves por metro quadrado. Sendo assim, em um limite de $39 \mathrm{~kg} / \mathrm{m}^{2}$ podem ser alocadas 15 aves com $2,6 \mathrm{~kg}$ ou 22 aves com $1,7 \mathrm{~kg}$ de peso vivo final. Essa é uma questão de bem-estar importante no cenário nacional, principalmente no caso de produção de fêmeas leves em grande escala, as quais são abatidas com peso vivo final de aproximadamente $1,5 \mathrm{~kg}$.

Dessa forma, este trabalho teve por objetivo avaliar os impactos da redução de densidades de lotação sobre o bem-estar de fêmeas leves, produzidas em aviário convencional do Sul do Brasil, tendo como indicadores variáveis fisiológicas, clínicas, comportamentais e índices zootécnicos.

\section{MATERIAL E MÉTODOS}

Este projeto foi aprovado pela Comissão de Ética no Uso de Animais da Instituição, protocolo número 003/2009. Dezoito mil pintinhos de um dia, da linhagem Cobb $500^{\circledR}$, foram alojados em um aviário semiclimatizado, com nebulizador e ventiladores. Aos 12 dias de idade, foram selecionadas 800 fêmeas com pesos semelhantes $(320 \pm 0,03 \mathrm{~g})$ e boas condições de saúde, as quais foram aleatoriamente alojadas em 16 baias de $4 \mathrm{~m}^{2}$, instaladas no centro do aviário. Usaram-se dois tratamentos e oito repetições. Nessas baias, foi respeitada a mesma relação de equipamentos por ave, 40 aves/comedouros e 12 aves/bebedouros. Considerando-se o peso final de $1,7 \mathrm{~kg} /$ ave, foram alojadas aves em densidade de 10 aves $/ \mathrm{m}^{2}$ (DL10) e 15 aves $/ \mathrm{m}^{2}$ (DL15), ou seja, $17 \mathrm{~kg} / \mathrm{m}^{2}$ e $25,5 \mathrm{~kg} / \mathrm{m}^{2}$, respectivamente. As densidades alojadas não excederam os limites em $\mathrm{kg} / \mathrm{m}^{2}$ ou em aves $/ \mathrm{m}^{2}$ estipulados pela RSPCA.

A temperatura e a umidade foram aferidas a cada hora utilizando-se aparelhos termo-higrômetros instalados a $60 \mathrm{~cm}$ de altura em relação à cama dos frangos. Após obtenção dos dados, foram calculados os índices de temperatura e umidade (ITU), conforme descrito por Thom (1959). Para a avaliação da porcentagem de tempo em que as aves ficaram submetidas a um ambiente fora da zona de conforto ambiental, foram utilizados os índices propostos pelo mesmo autor, segundo os quais ITU menor que 74 configura situação de conforto térmico; entre 74 e 79 representa situação de alerta; entre 79 e 84 indica situação de perigo; e acima de 84 configura situação de emergência.

Aos 31 dias de idade, uma baia de cada tratamento foi escolhida, de forma aleatória, para filmagem, por um período de 24 horas. A cada 30 minutos, o vídeo foi parado e o 
comportamento de cada animal da baia foi observado para construção do etograma. Os comportamentos observados nas aves foram: acocoradas, trocando de posição, comendo, bebendo, em pé, em pé próximo ao comedouro tubular e limpando as penas. Para definir movimentos duvidosos, o vídeo foi avançado 20 segundos, quadro a quadro, quando necessário.

No período matutino do $32^{\circ}$ dia de idade das aves e após restrição alimentar de uma hora, foram colhidos $2 \mathrm{~mL}$ de sangue (amostra heparinizada) de 16 aves por tratamento para realização de hemograma. As contagens totais de eritrócitos e leucócitos foram realizadas manualmente em hematocitômetros de Neubauer com sangue diluído em azul de toluidina a 0,01\%. A concentração de hemoglobina foi medida pelo método da cianometamoglobina e uma alíquota de sangue foi centrifugado em micro-hematócito a 2500rpm por cinco minutos, para determinação do volume globular. Para a diferenciação dos leucócitos, foram realizados esfregaços sanguíneos das amostras coletadas, imediatamente após a coleta. Essas lâminas foram coradas pela técnica de Wright, e 100 células foram analisadas em microscópio óptico com aumento de 1000 vezes. Desta análise foi determinada a relação entre heterófilo e linfócito (H:L), de acordo com Onbasilar e Aksoy (2005).

De outras 24 aves por tratamento, foram coletados $2 \mathrm{~mL}$ de sangue sem anticoagulante para realização dos testes bioquímicos de triglicerídeos, glicose, proteína total e albumina total, sendo determinadas utilizando-se kits comerciais (In Vitro-Human ${ }^{\circledR}$ ). As amostras foram centrifugadas e resfriadas $\left(4^{\circ} \mathrm{C}\right)$ para envio ao laboratório, no qual ficaram congeladas ($20^{\circ} \mathrm{C}$ ) até a análise. Após resultados de proteína total e albumina total, por subtração simples, foi obtido o nível de globulina de cada amostra.

As aves foram pesadas aos 12 e aos 33 dias de idade, para avaliação do desempenho zootécnico, considerando-se consumo de ração, ganho de peso e conversão alimentar. Aos 33 dias de idade, 10 aves por baia, em um total de 160 aves, foram selecionadas de forma aleatória para avaliação da habilidade de locomoção, sendo utilizado o método de escore de andadura, descrito por Jones et al. (2005). As aves caminharam por, no mínimo, 10 passos e foram classificadas de acordo com a dificuldade de locomoção, dividida em três níveis: zero para o frango sem problemas locomotores; um para aves caminhando de forma irregular, descompassadas e desbalanceadas; e dois para as aves com relutância para se mover, incapazes de caminhar muitos passos antes de se sentar. Os coxins plantares das mesmas 160 aves utilizadas para o escore de andadura foram avaliados em relação à pododermatite, seguindo a técnica semelhante à descrita por Jones et al. (2005), convencionando-se ausente para aves sem lesão ou com lesões iniciais e presente para aves com lesões maiores que $5 \mathrm{~mm}$.

Foram utilizadas estatística paramétrica para características de desempenho e locomoção e fisiológicas (teste t) e não paramétrica para indicadores comportamentais (Mann Whitney), com auxílio do pacote estatístico SAS.

\section{RESULTADOS E DISCUSSÃO}

O ITU apresentou variação acima do esperado durante o período do experimento. Observou-se que em 43,6\% do tempo os índices permaneceram acima do limite de conforto. Dawkins et al. (2004) citaram alta correlação entre o aparecimento de problemas de saúde com a porcentagem de tempo em que a temperatura e a umidade permaneceram fora da zona de conforto, evidenciando que condições ambientais inadequadas influenciam significativamente na diminuição do grau de bem-estar de frangos de corte.

Não foram observadas alterações significativas em nenhum dos comportamentos avaliados. Segundo Estevez et al. (1997), o aumento da densidade reduz o tempo gasto pelos frangos caminhando. Weeks et al. (2000) observaram diferenças entre frangos saudáveis e com problemas locomotores, os quais passavam $76 \%$ e $86 \%$ do tempo, respectivamente, acocorados. Apesar de esses estudos sustentarem a associação do aumento de densidade ou de problemas locomotores e a preferência em permanecer acocorado, tal fato não foi observado neste estudo (Tab. 1). 
Tabela 1. Mediana e valores máximos e mínimos dos comportamentos de frangas de corte, observados no $31^{\circ}$ dia de idade, submetidas a diferentes densidades de lotação

\begin{tabular}{|c|c|c|c|c|c|c|c|}
\hline \multirow[b]{2}{*}{ Período } & \multirow[b]{2}{*}{ Comportamento } & \multicolumn{3}{|c|}{$\begin{array}{c}10 \text { aves } / \mathrm{m}^{2} \\
\left(16,9 \mathrm{~kg} / \mathrm{m}^{2}\right)\end{array}$} & \multicolumn{3}{|c|}{$\begin{array}{c}15 \text { aves } / \mathrm{m}^{2} \\
\left(25,5 \mathrm{~kg} / \mathrm{m}^{2}\right)\end{array}$} \\
\hline & & $\begin{array}{c}\text { Mediana } \\
(\%)\end{array}$ & $\begin{array}{c}\text { Máximo } \\
(\%)\end{array}$ & $\begin{array}{c}\text { Mínimo } \\
(\%)\end{array}$ & $\begin{array}{c}\text { Mediana } \\
(\%)\end{array}$ & $\begin{array}{c}\text { Máximo } \\
(\%)\end{array}$ & $\begin{array}{c}\text { Mínimo } \\
(\%)\end{array}$ \\
\hline \multirow{6}{*}{ Noite } & Acocorados & 92,5 & 100 & 27,1 & 96,6 & 100 & 40,2 \\
\hline & Trocando de posição & 0 & 11,3 & 0 & 1,6 & 16,3 & 0 \\
\hline & Comendo & 0 & 30 & 0 & 0 & 26,8 & 0 \\
\hline & Bebendo & 0 & 11,6 & 0 & 0 & 7,4 & 0 \\
\hline & Em pé & 0 & 18,3 & 0 & 0 & 24,3 & 0 \\
\hline & Limpando penas & 0 & 4,6 & 0 & 0 & 2,4 & 0 \\
\hline \multirow{6}{*}{ Dia } & Acocorados & 67,4 & 97,5 & 36,9 & 62,5 & 90 & 29,7 \\
\hline & Trocando de posição & 0 & 6,5 & 0 & 3,2 & 16,6 & 0 \\
\hline & Comendo & 12,1 & 26,8 & 0 & 11,1 & 24,2 & 1,6 \\
\hline & Bebendo & 2,5 & 7,5 & 0 & 4,8 & 10 & 0 \\
\hline & Em pé & 4,7 & 18,3 & 0 & 3,2 & 21,6 & 0 \\
\hline & Limpando penas & 4,8 & 12,5 & 0 & 3,1 & 22,5 & 0 \\
\hline
\end{tabular}

Em relação aos valores de eritrócitos, hematócrito e hemoglobina, não foi encontrada diferença significativa entre os tratamentos (Tab. 2), sendo que esses dados são semelhantes aos encontrados por Tabeli et al. (2005) para a linhagem Cobb-500 ${ }^{\circledR}$, com índices de eritrócitos e hemoglobina de $2,17 \pm 0,1 \times 10^{6} / \mu \mathrm{L}$ e $13,48 \pm 0,2 \mathrm{~g} / \mathrm{dL}$, respectivamente.

No leucograma, uma diferença significativa pode ser observada entre os tratamentos em relação aos níveis de basófilos (Tab 2). Segundo Maxwel (1993), ao contrário do índice H:L que varia com níveis baixos a moderados de estresse, o aumento de basófilos está ligado a estresse extremo. Altan et al. (2000) verificaram um aumento no número de basófilos em frangos submetidos a uma temperatura de $39^{\circ} \mathrm{C}$ por uma hora, aumentando de 2,3 para 5,5\%. Neste trabalho, foram observados índices semelhantes aos frangos submetidos a estresse térmico, podendo ser um indicativo de estresse em ambos os tratamentos, porém a diferença estatística apontada não pode ser explicada pelos resultados de Maxwel (1993) e de Altan (2000), uma vez que todos os animais estavam submetidos ao mesmo ambiente. Sugerem-se maiores estudos relacionados ao comportamento das taxas de basófilo em frangos de corte.

Em relação às demais células brancas, não foram observadas diferenças significativas entre os tratamentos. Segundo Bounous e Stedman (2000), a variação normal de leucócitos está entre 12.000 e 30.000 células/ $\mu \mathrm{L}$ e a de linfócitos entre 7.000 e 17.500 células/ $\mu \mathrm{L}$, sendo relatada uma relação de linfócitos superior a $50 \%$ dos leucócitos totais (Tabeli et al., 2005). Neste experimento, ambos os tratamentos apresentam valores inferiores para leucócitos e para a relação entre linfócitos e leucócitos (Tab. 2). Esse fato pode ser um indicativo do aumento de estresse com uma consequente queda de linfócitos circulantes e aumento do índice de H:L. Para Gross e Siegel (1993), o índice de H:L pode ser considerado o mais sensível indicador de estresse crônico, sendo que índices acima de 0,7 indicam estresse moderado. Onbasilar e Aksoy (2005), em estudo com galinhas de postura criadas em gaiolas e em diferentes densidades, verificaram aumento de 0,62 para 0,95 no índice H:L, correlacionado ao aumento na concentração de corticosterona plasmática, que aumentou de 1,65 para $1,93 \mathrm{ng} / \mathrm{mL}$. Levando-se em consideração esses resultados, pode-se supor que as aves de ambos os tratamentos encontravam-se sob estresse, sendo uma possível consequência do tempo em que essas aves ficaram sem o devido conforto térmico ou uma interação entre densidade e a condição do ambiente, na qual o número de aves analisadas não permitiu sensibilidade suficiente para diferenças estatísticas significativas. 
Tabela 2. Variações na contagem de eritrócitos, hematócrito, hemoglobina, leucócitos totais, heterófilos, basófilos, eosinófilos, linfócitos, monócitos e relação de heterófilo:linfócito (H:L) de frangas de corte com 32 dias de idade, submetidas a diferentes densidades de lotação

\begin{tabular}{lcc}
\hline Característica fisiológica & $10 \mathrm{aves} / \mathrm{m}^{2}$ & $15 \mathrm{aves} / \mathrm{m}^{2}$ \\
$\left(16,9 \mathrm{~kg} / \mathrm{m}^{2}\right)$ & $\left(25,5 \mathrm{~kg} / \mathrm{m}^{2}\right)$ \\
\hline Eritrócitos $\left(10^{6} / \mu \mathrm{L}\right)$ & $2,02 \pm 0,2 \mathrm{a}$ & $1,9 \pm 0,3 \mathrm{a}$ \\
Hematócito $(\%)$ & $26,9 \pm 2,1 \mathrm{a}$ & $27 \pm 2,2 \mathrm{a}$ \\
Hemoglobina $(\mathrm{g} / \mathrm{dL})$ & $12,8 \pm 1,5 \mathrm{a}$ & $12,5 \pm 1,5 \mathrm{a}$ \\
Leucócitos $(/ \mu \mathrm{L})$ & $8875 \pm 4031 \mathrm{a}$ & $8125 \pm 3774 \mathrm{a}$ \\
Heterófilos $(\%)$ & $50,5 \pm 7,2 \mathrm{a}$ & $56,8 \pm 13,5 \mathrm{a}$ \\
Basófilos $(\%)$ & $6,3 \pm 2,3 \mathrm{a}$ & $4,5 \pm 1,8 \mathrm{~b}$ \\
Eosinófilos $(\%)$ & $0 \mathrm{a}$ & $0,1 \pm 0,3 \mathrm{a}$ \\
Linfócitos $(\%)$ & $40,7 \pm 8,8 \mathrm{a}$ & $36,3 \pm 12,7 \mathrm{a}$ \\
Monócitos $(\%)$ & $2,5 \pm 1,4 \mathrm{a}$ & $2,0 \pm 0,8 \mathrm{a}$ \\
H:L & $1,3 \pm 0,4 \mathrm{a}$ & $1,9 \pm 1,0 \mathrm{a}$ \\
\hline
\end{tabular}

Letras diferentes na mesma linha indicam diferença significativa entre as médias $(\mathrm{P}<0,05)$.

Observou-se diferença na concentração de triglicerídeos, com valores mais altos nas aves da maior densidade (Tab. 3). Concentrações altas de triglicerídeos e glicose podem ser consideradas indicadores de estresse agudo, pois são prontamente disponibilizadas pelo sistema nervoso simpático, para serem utilizados em situações adversas. De acordo com Puvadolpirod e Thaxton (2000b), em animais que receberam aplicações diárias de hormônio adrenocorticotrófico (8IU/kg de peso vivo/dia), o qual possui efeito sobre o sistema simpático, ocorre até o quarto dia pós-administração um aumento pronunciado de triglicerídeos e glicose, sendo que aos sete dias se observa uma queda desses níveis, indicando uma tentativa de adaptação das aves ao estresse. Em seu estudo, o nível de triglicerídeo, inicialmente em $81,2 \mathrm{mg} / \mathrm{dL}$, teve um pico de 164,1 e posterior queda para $121,4 \mathrm{mg} / \mathrm{dL}$, e o de glicose passou de 247 para 878 e $379 \mathrm{mg} / \mathrm{dL}$. Quando os dados de triglicerídeos desse experimento são comparados com os obtidos por Puvadolpirod e Thaxton (2000b), pode-se perceber que, em ambas as densidades, os valores estão acima dos valores basais. Porém, neste experimento, foi observada uma diferença significativa de triglicerídeos em animais produzidos em maior densidade, sendo um indicativo de enfrentamento de maiores desafios pelos animais em maior densidade.

Os níveis de proteína e albumina total não apresentaram diferenças significativas entre os tratamentos. Porém, observa-se uma diferença significativa do nível de globulina sérica em animais produzidos em maior densidade (Tab. 3). Tais resultados diferem daqueles de El-Deek e Al-Harthi (2004), que não observaram diferença em frangos produzidos até os 43 dias de idade em densidades de 10 e 14 aves $/ \mathrm{m}^{2}$. Neste experimento, pode-se associar a diferença de globulina a uma possível diferença na produção de imunoglobulinas, uma vez que o nível de linfócitos encontra-se abaixo dos valores de referência, porém uma discussão mais detalhada demanda a determinação do perfil eletroforético.

Tabela 3. Média e desvio-padrão de triglicerídeos, glicose, proteína, albumina, globulina, relação entre peso de fígado e peso vivo e entre baço e peso vivo, em frangas de corte com 32 dias de idade, submetidas a diferentes densidades de lotação

\begin{tabular}{lcc}
\hline \multirow{2}{*}{ Característica fisiológica } & $10 \mathrm{aves} / \mathrm{m}^{2}$ & $15 \mathrm{aves} / \mathrm{m}^{2}$ \\
\cline { 2 - 3 } & $\left(16,9 \mathrm{~kg} / \mathrm{m}^{2}\right)$ & $\left(25,5 \mathrm{~kg} / \mathrm{m}^{2}\right)$ \\
\hline Triglicerídeos $(\mathrm{ng} / \mathrm{dL})$ & $158,9 \pm 32,9 \mathrm{~b}$ & $186,3 \pm 43,9 \mathrm{a}$ \\
Glicose $(\mathrm{mg} / \mathrm{dL})$ & $236,1 \pm 13,2 \mathrm{a}$ & $231,2 \pm 13,2 \mathrm{a}$ \\
Proteína Total $(\mathrm{g} / \mathrm{dL})$ & $3,9 \pm 0,6 \mathrm{a}$ & $3,6 \pm 0,4 \mathrm{a}$ \\
Albumina $(\mathrm{g} / \mathrm{dL})$ & $2,1 \pm 0,2 \mathrm{a}$ & $2,1 \pm 0,2 \mathrm{a}$ \\
Globulina $(\mathrm{g} / \mathrm{dL})$ & $1,8 \pm 0,5 \mathrm{a}$ & $1,5 \pm 0,4 \mathrm{~b}$ \\
\hline
\end{tabular}

Letras diferentes na mesma linha indicam diferença significativa entre as médias $(\mathrm{P}<0,05)$. 
Nos índices de desempenho, foi observada diferença significativa em relação ao peso médio final (Tab. 4). Resultados diferentes para peso final foram citados por Dozier et al. (2005), que não observaram diferenças no peso vivo de frangos com 32 dias de idade, produzidos em sistemas climatizados nas densidades de 9, 11, 12 e 13 aves $/ \mathrm{m}^{2}$. Moreira et al. (2004) estudaram, em aviários convencionais, o efeito das densidades de 10,13 e 16 aves $/ \mathrm{m}^{2}$ em frangos produzidos até 35 dias de idade e observaram queda significativa no ganho de peso entre as densidades de 10 e 16 aves $/ \mathrm{m}^{2}$. Segundo Dawkins et al. (2004), os impactos da alta densidade sobre o bem-estar não podem ser comparados de forma simplista, sem ser considerada a qualidade ambiental. Dessa forma, pode-se especular que a diminuição da qualidade ambiental, gerada pela falta de controle efetivo sobre condições ambientais neste experimento, associada ao aumento da densidade provocaram efeito sinérgico na redução do desempenho em DL15, sendo um indicativo de menor grau de bem-estar neste tratamento.

Tabela 4. Consumo médio de ração por ave, ganho de peso médio diário, peso médio final e conversão alimentar ajustada na fase de frangas de corte submetidas a diferentes densidades de lotação, avaliados de 12 a 33 dias de idade

\begin{tabular}{lcc}
\multicolumn{1}{c}{$\begin{array}{c}\text { Característica de } \\
\text { desempenho }\end{array}$} & $10 \mathrm{aves} / \mathrm{m}^{2}$ & $15 \mathrm{aves} / \mathrm{m}^{2}$ \\
& $16,9 \mathrm{~kg} / \mathrm{m}^{2}$ & $25,5 \mathrm{~kg} / \mathrm{m}^{2}$ \\
\hline Consumo médio de ração/ave $(\mathrm{kg})$ & $2,22 \pm 0,01$ & $2,16 \pm 0,03$ \\
Ganho de peso médio diário (kg/dia) & $0,085 \pm 0,01$ & $0,081 \pm 0,07$ \\
Peso médio final $(\mathrm{kg})$ & $1,69 \pm 0,22 \mathrm{a}$ & $1,65 \pm 0,10 \mathrm{~b}$ \\
Conversão alimentar ajustada na fase & $1,62 \pm 0,07$ & $1,61 \pm 0,03$
\end{tabular}

Letras diferentes na mesma linha indicam diferença significativa entre as médias $(\mathrm{P}<0,05)$.

Nesse trabalho, os escores de andadura e pododermatite não diferiram entre os tratamentos (Tab. 5). Os dados de escore de andadura foram semelhantes aos encontrados por Sorensen et al. (2000), não sendo observada diferença, em frangos com 28 dias de idade, quando se aumentou a densidade de 10 para 16 aves $/ \mathrm{m}^{2}$. Em relação aos problemas de pododermatite, o maior número de indivíduos com pododermatite em DL15, apesar de não significativo, talvez esteja relacionado ao aparecimento de maior variação no ganho de peso diário e menor peso final.

Como padrões para serem seguidos nas avaliações de bem-estar, Grandin (2009) estabelece porcentagens médias de aves livres de problemas que causem queda no grau de bemestar. Seguindo o mesmo método de avaliação para escore de andadura descrito neste trabalho, com três graus, a autora estabelece que $72,6 \%$ das aves não devem apresentar alterações de locomoção, considerando os melhores lotes aqueles com $95 \%$ das aves capazes de andar pelo menos dez passos. As observações indicam que as aves de ambos os tratamentos apresentam uma condição superior ao limite estipulado, estando o DL15 classificado como um lote de ótimo escore de andadura. Tal resultado sugere uma condição superior das aves estudadas no que tange ao indicador escore de andadura relativo à literatura internacional. Adicionalmente, os valores numericamente melhores do grupo DL15 podem estar assciados ao menor peso corporal dessas aves em relação ao grupo DL10. Em se tratando do índice de pododermatite, $81,2 \%$ de aves livres de lesões de patas foram utilizados por Grandin (2005) para estipular o limite mínimo de um lote considerado normal para os padrões de bem-estar animal. Neste experimento, o DL10 apresenta-se acima do limite mínimo, porém o DL15 está com índice pior ao indicado por Grandin (2009), fortalecendo, assim, a ideia de associação entre aumento do índice de pododermatite e queda de desempenho. 
Tabela 5. Índices de escore de andadura e de pododermatite de frangas de corte com idade de 33 dias de idade, submetidas a diferentes densidades de lotação

\begin{tabular}{|c|c|c|c|c|}
\hline \multirow[t]{2}{*}{ Variáveis } & \multicolumn{2}{|c|}{$\frac{10 \text { aves } / \mathrm{m}^{2}}{\left(16,9 \mathrm{~kg} / \mathrm{m}^{2}\right)}$} & \multicolumn{2}{|c|}{$\frac{15 \text { aves } / \mathrm{m}^{2}}{\left(25,5 \mathrm{~kg} / \mathrm{m}^{2}\right)}$} \\
\hline & Mediana & Máx - Mín & Mediana & Máx - Mín \\
\hline \multicolumn{5}{|l|}{ Escore de andadura } \\
\hline Grau $0(\%)$ & 90 & $90-70$ & 100 & $100-90$ \\
\hline Grau $1(\%)$ & 10 & $15-10$ & 0 & $10-0$ \\
\hline Grau $2(\%)$ & 0 & 0 & 0 & 0 \\
\hline \multicolumn{5}{|l|}{ Pododermatite } \\
\hline Ausente (\%) & 85 & $90-77$ & 80 & $80-70$ \\
\hline Presente (\%) & 15 & $22-10$ & 20 & $30-20$ \\
\hline
\end{tabular}

\section{CONCLUSÕES}

A consideração conjunta de menor peso final, maiores taxas de triglicerídeos e menores valores de globulinas sugere menor grau de bem-estar em DL15, havendo, assim, um impacto positivo na redução de 15 para 10 aves $/ \mathrm{m}^{2}$ na produção de fêmeas para abate precoce. Os valores acima do recomendado para a espécie do índice de temperatura e umidade e a alta relação entre heterófilo:linfócito sugerem baixo grau de bemestar em ambas as densidades.

\section{REFERÊNCIAS BIBLIOGRÁFICAS}

ALTAN, O.; ALTAN, A.; ÇABUK, M. et al. Effects of heat stress on some blood parameters in broilers. Turk J. Vet. Anim. Sci.. v.24, p.145$148,2000$.

BESSEI, W. Welfare of broilers: a review. World's Poult. Sci. J., v.62, p.455-466, 2006.

BOUNOUS, D.I.; STEDMAN, N.L. Normal avian heamtology: chicken and turkey. In: FELDMAN, B.F.; ZINKL, J.G.; JAIN, N.C. Schalm's veterinary hematology, 5.ed. Philadelphia: Lippincott Williams \& Wilkins, 2000. p.417-432.

DAWKINS, M.S.; DONNELLY, C.A.; JONES, T.A. Chicken welfare is influenced more by housing conditions than by stocking density. Nature, v.427, p.342-343, 2004.

DOZIER III, W.A.; THAXTON J.P.; BRANTON S.L. et al. Stocking density effects on growth performance and processing yields of heavy broilers. Poult. Sci., v.84, p.1332-1338, 2005.
EL-DEEK, A.A.; AL-HARTHI, M.A. Responses of modern broiler chicks to stocking density, green tea, commercial multi enzymes and their interactions on productive performance, carcass characteristics, liver composition and plasma constituents. Int. J. Poult. Sci., v.3, p.635-645, 2004.

ESTEVEZ, I.; NEWBERRY, R.C.; ARIAS DE REYNA, L. Broiler chickens: A tolerant social system? Etologia, v.5, p.19-29, 1997.

ESTEVEZ, I. Density allowances for broilers: where to set the limits? Poult. Sci., v.86, p.12651272, 2007.

GRANDIN, T. Poultry slaughter plant and farm audit: critical control points for bird welfare, (2009) Disponível em: <http//www.grandin. com/poultry.audit.html> Acesso em 20/01/2010.

GROSS, W.B.; SIEGEL, H.S. General principles of stress and welfare, In: GRANDIN, T. Livestock handling and transport wallingford: CAB Internacional, 1993. Cap.3, p.21-34.

JONES, T.A.; DONNELLY, C.A.; DAWKINS, M.S. Environmental and management factors affecting the welfare of chickens on commercial farms in the United Kingdom and Denmark stocked at five densities. Poultr. Sci., v.84, p.1155-1165, 2005.

MARTRENCHAR, A.; MORISSE, J.P.; HUONNIC, D. et al. Influence of stocking density on some behavioural, physiological and productivity traits of broilers. Vet. Res., v.28, p.473-480, 1997.

MAXWELL, M.H. Avian blood leucocyte responses to stress. World's Poutr. Sci. J., v.49, p.34-43, 1993. 
MOREIRA, J.; MENDES, A.A.; ROÇA, R.O. et al. Efeito da densidade populacional sobre desempenho, rendimento de carcaça e qualidade da carne em frangos de corte de diferentes linhagens comerciais. Rev. Bras. Zootec., v.33, p.1506-1519, 2004.

ONBASILAR, E.E.; AKSOY, F.T. Stress parameters and immune response of layer under different cage floor and density conditions. Livest. Prod. Sci., v.95, p.255-263, 2005.

POULTRY standards, 2009-2010. Disponível em: http://www.assuredchicken.org.uk/resources/000 /315/869/ACP-Standards_2009-2010.pdf, Acesso em: 16/06/09.

PROTOCOLO de bem-estar de frangos e perus. 2008. Disponível em:

<http://www.uba.org.br/protocolo_de_bemestar_para _frangos_e_perus_14_07_08.pdf $\rangle$. Acessado em 16 jun 09.

PUVADOLPIROD, S.; THAXTON, J.P. Model of physiological stress in chickens 1 . Response parameters. Pout. Sci., v.79, p.363-369, 2000.

PUVADOLPIROD, S., THAXTON, J.P., Model of physiological stress in chickens 3. Temporal patterns of response, Poult. Sci., v.79, p.377-382, 2000b.
RSPCA welfare standards for chickens. 2008. Disponível em: <http://www.rspca.org.uk/servlet/ BlobServer?blobtable $=$ RSPCABlob\&blobcol=urlblob \&blobkey=id\&blobheader=application/pdf\&blobwher $\mathrm{e}=1158755026986>$. Acessado em: 04/05/2008.

SORENSEN, P.; SU, G.; KESTIN, S.C. Effects of age and stocking density on leg weakness in broiler chickens. Poult. Sci., v.79, p.864-870, 2000.

TABELI, A.; ASRI-REZAEI, S.; ROZEHCHAI, R. et al. Comparative studies on haematological values of broiler strains (Ross, Cobb, Arbor-acres and Arian), Int. J. Poult. Sci., v.4, p.573-579, 2005.

THOM, E.C. The discomfort index. Weatherwise, v.12, p.57-60, 1959.

WEEKS, C.A.; DANBURY, T.C.; DAVIES, H.C. et al. The behavior of broiler chickens and its modification by lameness. Appl. Anim. Behav. Sci., v.67, p.111-125, 2000. 